\title{
Using a Bessel light beam as an ultrashort period helical undulator
}

\author{
B. C. Jiang, ${ }^{1}$ Q. L. Zhang, ${ }^{1}$ J. H. Chen, ${ }^{1}$ and Z. T. Zhao ${ }^{1,2^{*}}$ \\ ${ }^{1}$ Shanghai Institute of Applied Physics, Chinese Academy of Sciences, Shanghai 201800, China \\ ${ }^{2}$ ShanghaiTech University, Shanghai 201210, China \\ (Received 2 January 2017; published 24 July 2017)
}

\begin{abstract}
An undulator is a critical component to produce synchrotron radiation and a free electron laser. When a Bessel light beam carrying the orbit angular momentum copropagates with an electron beam bunch, a net transverse deflection force will be subjected to the latter one. As a result of dephasing effect, the deflection force will oscillate and act as an undulator. For such a laser based undulator, the period length can reach submillimeter level, which will greatly reduce the electron energy for the required $\mathrm{x}$-ray production.
\end{abstract}

DOI: $10.1103 /$ PhysRevAccelBeams.20.070701

\section{INTRODUCTION}

A magnetostatic undulator is in periodic structures of dipole magnets $[1,2]$. The static magnetic field of the undulator is perpendicular to the electron beam trajectory, and periodically changes its directions, which causes an electron beam bunch to follow an undulating trajectory, hence the energy radiations. The radiation brightness from an undulator at the resonance wavelength is $N^{2}$ times higher than that from a single bending magnet, where $N$ is total period number of the undulator.

The radiation wavelength can be calculated by Eqs. (1) and (2) $[2,3]$ :

$$
\begin{aligned}
\lambda_{\mathrm{rad}} & =\frac{\lambda_{u}}{2 \gamma^{2}}\left(1+\frac{K^{2}}{2}+\gamma^{2} \theta^{2}\right), \\
K & =\frac{e B \lambda_{u}}{2 \pi m_{e} c}=0.934 B(T) \lambda_{u}(\mathrm{~cm}),
\end{aligned}
$$

where, $\lambda_{u}$ is period length of the undulator; $B$ is peak magnetic field of the undulator; $\lambda_{\text {rad }}$ is the radiation wavelength; $\theta$ is the radiation angle; $\gamma, e$ and $m_{e}$ are the Lorentz factor, charge and rest mass of electron, respectively; and $c$ is the speed of light.

An important direction of the undulator improvement is to decrease the period length. The shorter the period length of the undulator, the lower the electron energy required for a desired $\mathrm{x}$ ray, hence a great reduction of the facility scale and cost.

For a practical configuration, the $K$ value of an undulator should be in order of 1 . To this end, the shorter the period length, the higher the peak magnetic field should be.

\footnotetext{
Corresponding author. zhaozt@sinap.ac.cn

Published by the American Physical Society under the terms of the Creative Commons Attribution 4.0 International license. Further distribution of this work must maintain attribution to the author(s) and the published article's title, journal citation, and DOI.
}

This prevents the undulator period from being ultrashort. The in-vacuum undulator was developed for short period approach $[4,5]$. In this type of undulator, the permanent magnets are installed in a vacuum tank, thus the undulator pole gap can be much smaller, the peak field can be increased, and the period length can be reduced.

The discovery of increasing remanent field and coercivity of the permanent magnet at low temperatures provides the possibility of building the cryogenic permanent magnet undulator [6,7] with a shorter period, a little bit, though, at the cost of an additional liquid nitrogen cryogenic system. The superconducting technology helps to build undulators of even shorter periods [8,9]. However, even for the state-ofthe-art technology, the period length of a magnetostatic undulator is beyond $1 \mathrm{~mm}$ [10]. It is possible for an $\mathrm{rf}$ undulator $[11,12]$ to achieve period length of shorter than $1 \mathrm{~mm}$. However, for lacking of a high power THz source, it is still hard to realize a millimeter-period undulator.

The optical undulator has been proposed for compact FEL purpose for years. When an intense and long enough laser pulse counterpropagates with the electron beam, the laser may act as an undulator [13-16]. The period length of the optical undulator is in the micron range. This requires that the electron beam is orders of magnitude brighter than the existing electron source for FEL generation. The laser plasma undulator $[16,17]$ was just proposed to build the submillimeter period undulator, but the fact that the electrons do not pass through free space may prevent its use in storage rings.

In another area, the light beam carrying orbital angular momentum (OAM) discovered in the early 1990s in Laguerren-Gaussian beams $[18,19]$ has attracted myriad modern interests. Among them, trapping and accelerating charged particles is an amazing application [20-23]. For the Bessel beam [24], a more simple solution of the light wave in open free space carries OAM, the field can be separated into transverse and longitudinal parts. Being usually used to analyze trapping of charged particles, the Bessel beam shows the ability of wiggling the charged particles at low energies, while for its interaction with relativistic electrons, its properties are still unveiled. 


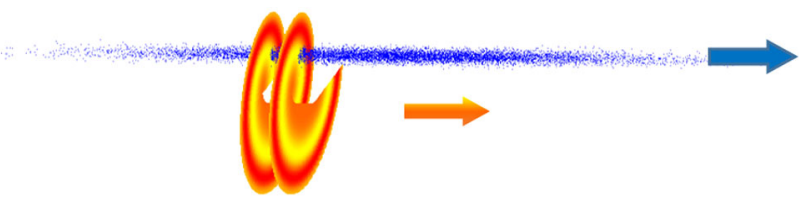

FIG. 1. Sketch of Bessel light beam (red-orange vortex) interaction with electron beam (blue dot).

In the following sections we will show that a Bessel light beam can be used for undulating relativistic electrons when it is copropagating as shown in Fig. 1. With the advent of a high power laser, it is possible to achieve a $K$ value of around 1 for the Bessel light beam undulator (BLU) with a period length approaching submillimeter. The interaction of the Bessel light beam with the relativistic electron beam is a supplement of laser Compton scattering (LCS) [25]. However for BLU, the interaction distance is much longer and higher flux can be produced comparing to LCS.

\section{TRANSVERSE FORCE OF BESSEL LIGHT BEAM TO THE RELATIVISTIC ELECTRON}

For a monochromatic Bessel light beam, in the dimensionless system $(c=1)$, the electric and magnetic fields of the wave in the Cartesian coordinate for paraxial approximation can be expressed as [26]

$$
\begin{gathered}
\left(\begin{array}{c}
\mathcal{E}_{x} \\
\mathcal{E}_{y} \\
\mathcal{E}_{z}
\end{array}\right)=\left(\begin{array}{c}
\boldsymbol{\kappa}_{-} \boldsymbol{C}_{M+1}+\boldsymbol{\kappa}_{+} \boldsymbol{C}_{M-1} \\
\boldsymbol{\kappa}_{-} \boldsymbol{S}_{M+1}-\boldsymbol{\kappa}_{+} \boldsymbol{S}_{\boldsymbol{M}-1} \\
2 \boldsymbol{S}_{\boldsymbol{M}}
\end{array}\right), \\
\left(\begin{array}{c}
\mathscr{B}_{\boldsymbol{x}} \\
\mathscr{B}_{\boldsymbol{y}} \\
\mathscr{B}_{z}
\end{array}\right)=\left(\begin{array}{c}
\boldsymbol{\kappa}_{-} \boldsymbol{S}_{\boldsymbol{M}+1}+\boldsymbol{\kappa}_{+} \boldsymbol{S}_{\boldsymbol{M}-1} \\
-\boldsymbol{\kappa}_{-} \boldsymbol{C}_{\boldsymbol{M}+1}+\boldsymbol{\kappa}_{+} \boldsymbol{C}_{\boldsymbol{M}-1} \\
2 \boldsymbol{C}_{\boldsymbol{M}}
\end{array}\right), \\
\boldsymbol{C}_{\boldsymbol{M}}=\cos \left(\boldsymbol{k}_{\|} z-\chi \omega t+\boldsymbol{M} \phi\right) \boldsymbol{J}_{\boldsymbol{M}}\left(\boldsymbol{k}_{\perp} \boldsymbol{\rho}\right), \\
\boldsymbol{S}_{\boldsymbol{M}}=\sin \left(\boldsymbol{k}_{\|} z-\chi \omega \boldsymbol{t}+\boldsymbol{M} \phi\right) \boldsymbol{J}_{\boldsymbol{M}}\left(\boldsymbol{k}_{\perp} \boldsymbol{\rho}\right),
\end{gathered}
$$

where $z$ is the light propagation direction, $\rho=\sqrt{\boldsymbol{x}^{2}+\boldsymbol{y}^{2}}$ is the transverse distance to the $z$ axis, $\phi$ is the azimuthal phase to the $z$ axis, $M$ is the order of Bessel beam, and

$$
\kappa_{ \pm}=\frac{k \pm \boldsymbol{k}_{\|}}{\boldsymbol{k}_{\perp}}
$$

In this paper, we only consider the forward propagated wave with $\chi=\mathbf{1}$. The wave numbers have the following relationships:

$$
\boldsymbol{k}=\sqrt{\boldsymbol{k}_{\|}^{2}+\boldsymbol{k}_{\perp}^{2}}
$$

$$
\omega=c k
$$

The Lorentz force in the horizontal $(x)$ and vertical $(y)$ planes for a copropagated relativistic electron $(v \cong c)$ can be derived by

$$
F_{x}(z, t, \rho, \phi)=-e\left(\mathcal{E}_{x}-c \mathscr{B}_{\boldsymbol{y}}\right)=-2 e \boldsymbol{\kappa}_{-} \boldsymbol{C}_{\boldsymbol{M}+1}(z, t, \rho, \phi),
$$

$F_{y}(z, t, \rho, \phi)=-e\left(\mathcal{E}_{\boldsymbol{y}}+c \mathscr{B}_{\boldsymbol{x}}\right)=-2 e \boldsymbol{\kappa}_{-} \boldsymbol{S}_{\boldsymbol{M}+1}(z, t, \rho, \phi)$.

Forces in $x$ and $y$ planes expressed in Eq. (8) oscillate in a $\sin$ waveform with phase difference of $\pi / 2$, forming a force like a helical undulator.

From Eqs. (4), (6) and (7), it can be found that the phase velocity of the Bessel beam is faster than the light speed. As the relativistic electron is in velocity of $v \cong c$, the phase slip of a relativistic electron to the light produces an up-conversion undulate period of the laser wavelength:

$$
\lambda_{u}=\frac{1}{1-\boldsymbol{k}_{\|} / \boldsymbol{k}} \lambda_{\text {laser }}
$$

The key factor for a Bessel light to undulate the relativistic electron is that its phase velocity is faster than the light speed. The magnetic field cannot be canceled completely by the electric field when it is seen by a copropagated relativistic electron, leaving a net periodic oscillated deflecting force. As the electric magnetic (EM) field of Bessel light rotates in the transverse plane, by neglecting energy absorption from the laser to the electrons, BLU only produces circular polarized radiations.

\section{DETERMINANTS OF THE UNDULATOR DEFLECTION PARAMETER $K$}

In this section we will analyze the undulator deflection parameter $K$. In the case of $\boldsymbol{k}_{\perp} \ll \boldsymbol{k}$, we have $\boldsymbol{\kappa}_{+} \gg \boldsymbol{\kappa}_{-}$. The transverse EM field expressed on the right-hand side of Eq. (3) is then mainly composed of the term with coefficient $\boldsymbol{\kappa}_{+}$such as

$$
\mathscr{B}_{\boldsymbol{y}} \approx \kappa_{+} \boldsymbol{C}_{M-1} .
$$

When an electron travels at a certain transverse place $\left(\rho_{0}, \phi_{0}\right)$ in the Bessel light beam, we have

$$
\left.\left.\mathscr{B}_{\boldsymbol{y}} \widehat{(z, t)}\right|_{\rho 0, \phi 0} \approx \boldsymbol{\kappa}_{+} \boldsymbol{C}_{\boldsymbol{M}-1} \widehat{(z}, t\right)\left.\right|_{\rho 0, \phi 0},
$$

where $\hat{B}$ denotes maximum value of the $\mathrm{B}$. The transverse force in the horizontal plane can be written as 


$$
\begin{aligned}
\left.F_{x}(z, t)\right|_{\rho 0, \phi 0} & =-\left.2 e \frac{\boldsymbol{\kappa}_{-}}{\boldsymbol{\kappa}_{+}} \boldsymbol{C}_{\boldsymbol{M}+1}(z, t)\right|_{\rho 0, \phi 0} \boldsymbol{\kappa}_{+} \\
& \approx-\left.e\left(1-\frac{\boldsymbol{k}_{\|}}{\boldsymbol{k}}\right) \boldsymbol{C}_{\boldsymbol{M}+1}(z, t)\right|_{\rho 0, \phi 0} \boldsymbol{\kappa}_{+} .
\end{aligned}
$$

Substituting Eq. (11) to Eq. (12) we get

$$
F_{x}(z, t) \approx-e\left(1-\frac{\boldsymbol{k}_{\|}}{\boldsymbol{k}}\right) \frac{\widehat{\mathscr{B}_{\boldsymbol{y}}(z, t)}}{\left.\boldsymbol{C}_{\boldsymbol{M}-1} \widehat{(z}, t\right)} \boldsymbol{C}_{\boldsymbol{M}+1}(z, t)
$$

The force of the laser is equivalent to a static magnet with peak value as

$$
\widehat{B_{y_{-} \mathrm{eff}}} \approx\left(1-\frac{\boldsymbol{k}_{\|}}{\boldsymbol{k}}\right) \frac{\left.\boldsymbol{C}_{\boldsymbol{M}+1} \widehat{\widehat{(z}}, t\right)}{\boldsymbol{C}_{\boldsymbol{M - 1}} \widehat{(z, t)}} \widehat{\mathscr{B}_{\boldsymbol{y}}(z, t)}
$$

Thus the undulator defection parameter $K_{x}$ is

$$
K_{x}=0.934 \widehat{B_{y_{-} \text {eff }}} \lambda_{u}=0.934 \times \frac{\left.C_{M+1} \widehat{(z}, t\right)}{\left.C_{M-1} \widehat{(z}, t\right)} \widehat{\mathscr{B}_{\boldsymbol{y}}(z, t)} \lambda_{\text {laser }} .
$$

The vertical plane can be treated in the same way (we will not repeat here).

For a circular polarized helical undulator the total defection parameter is

$$
K=\sqrt{2} K_{x}
$$

Without the factor of $\boldsymbol{k}_{\perp}$, the parameter $K$ of the BLU is just determined by the laser power density, the wavelength and the Bessel light beam order $M$. It is consistent with the rf and optical undulator. When degenerating Bessel light beam to a plane wave laser $\left(\boldsymbol{k}_{\perp}=\mathbf{0}\right)$, as it copropagates with the electron, $E$ field is almost canceled by the $B$ field, the Lorentz force is $B_{\text {eff }}=$ $(1-\beta) B_{y}$, while the electron dephasing in the light makes $\lambda_{u}=\lambda_{\text {laser }} /(1-\beta)$, where $\beta$ is the electron velocity in units of light speed. As the plane wave counterpropagates with the electron, the $E$ field adds up with the $B$ field, $B_{\text {eff }}=2 B_{y}$, and the undulator period $\lambda_{u}=\lambda_{\text {laser }} / 2$. For both cases, we have $K=B_{\text {eff }} \lambda_{\mathrm{u}}=0.934 \widehat{\mathscr{B}}_{y} \lambda_{\text {laser }}$.

\section{LASER POWER REQUIREMENT}

An ideal Bessel light beam has an infinitely extended transverse profile and carries infinite power, but Bessel light beams in practice cannot be ideal, with finite radius and power. A nonideal Bessel beam in radius $R$ has diffraction distance [22]
TABLE I. BLU parameters.

\begin{tabular}{lc}
\hline \hline Laser wavelength $/ \mu \mathrm{m}$ & \\
$\boldsymbol{k}_{\perp} / \boldsymbol{k}$ & 0.199 \\
$\boldsymbol{k}_{\|} / \boldsymbol{k}$ & 0.98 \\
$M$ & 2 \\
$\lambda_{u} / \mathrm{mm}$ & 0.53 \\
$R / \mathrm{mm}$ & 5 \\
$L / \mathrm{mm}$ & 25 \\
Periods & 47 \\
Laser power/TW & 9.3 \\
$K_{x}(\mathrm{~K})$ & $0.5(0.707)$ \\
\hline \hline
\end{tabular}

$$
L=R \frac{k}{\boldsymbol{k}_{\perp}}
$$

The Bessel light beam holds $N$ rings within radius $R$ and will be diffracted layer by layer until the innermost ring diffracts away at the end of diffraction distance.

For $k_{-} / k \ll 1$, the laser power is mainly determined by the EM term on the right-hand of Eq. (3) with the $\boldsymbol{\kappa}_{+}$coefficient. The power can be integrated approximately as

$$
P \approx 2 \boldsymbol{\kappa}_{+}^{2} \int_{0}^{2 \pi} \mathrm{d} \phi \int_{0}^{R} \rho J_{M-1}\left(\boldsymbol{k}_{\perp} \rho\right)^{2} d \rho .
$$

Equation (18) is in normalized form as it is directly derived from Eq. (3). For power calculation, a factor $c B_{0}^{2} / 2 \mu_{0}$ should be multiplied, where $B_{0}$ is the normalized magnetic field for Eq. (3), and $\mu_{0}$ is the permeability of vacuum.

Take a $\mathrm{CO}_{2}$ laser in wavelength of $10.6 \mu \mathrm{m}$ for example, to produce an undulator of 47 periods, $\lambda_{u}=0.53 \mathrm{~mm}$, and $K_{x}=0.5$, a laser power of $9.3 \mathrm{TW}$ is needed. The laser beam parameters are listed in Table I.

In Ref. [23] a laser carrying OAM at Terawatt (TW) level demonstrated the feasibility of building a BLU for x-ray production.

\section{BEAM TRACKING AND PHOTON FLUX}

Here we take the Shanghai soft x-ray linac beam [27] as an example for tracking, whose electron energy is $840 \mathrm{MeV}$. With BLU parameters listed in Table I, $1.2 \AA$ hard $\mathrm{x}$ ray will be produced.

To maximize the deflection force, it is nature to align the electron beam at the place where $\boldsymbol{J}_{\boldsymbol{M}+1}\left(\boldsymbol{k}_{\perp} \boldsymbol{\rho}\right)$ gets its peak value, and where the deflecting force has a small range of flattop in amplitude in the radial direction, which allows a relatively large part of an electron beam to radiate monochromatically.

In the tracking, both transverse and longitudinal parts of the EM fields are counted while radiation effect is neglected. The tracking is mainly focused on finding out 

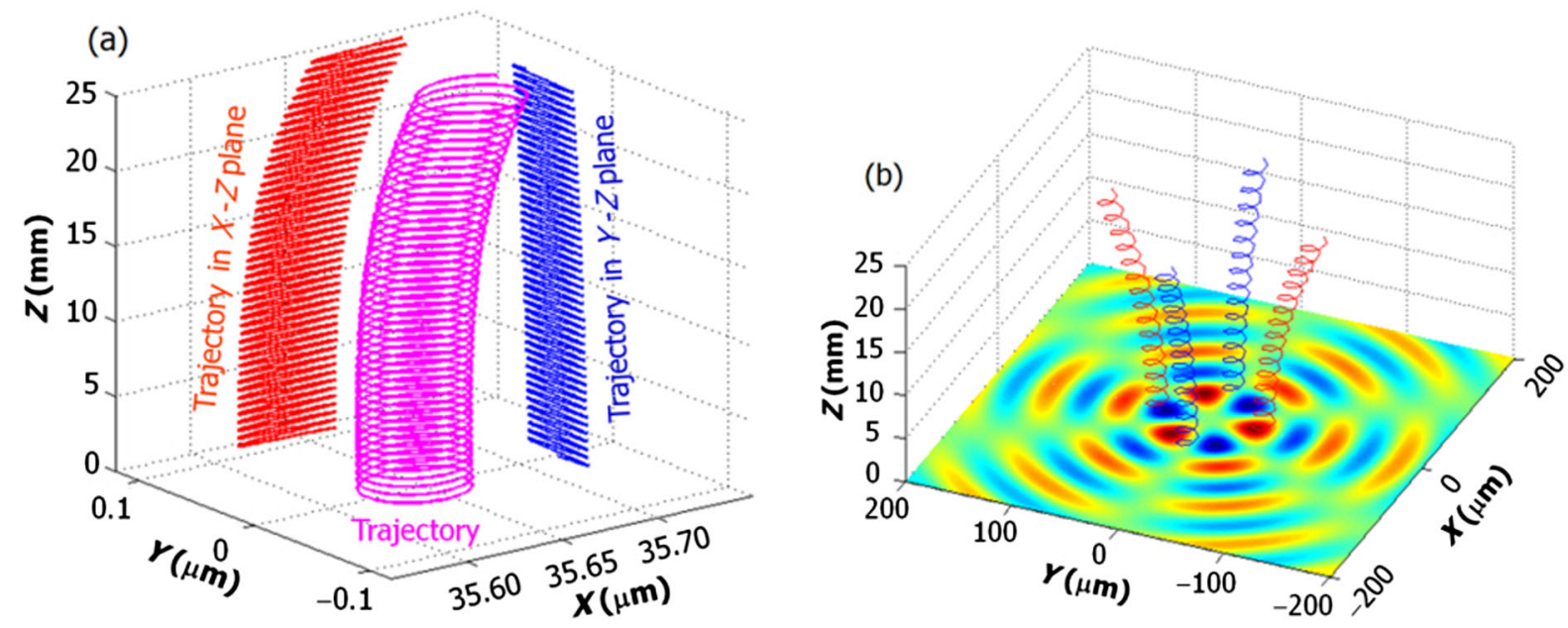

FIG. 2. Tracking result of electron trajectory (a), and the defocus effect (b), with the zoomed beam trajectory for a clear view, and with the electrical field rotating counterclockwise in the bottom.

the electron trajectory which can be used for undulator phase error evaluation.

In the tracking, it is found that the longitudinal electrical field causes an energy modulation of the electron beam, resulting in a deflecting effect in the radial direction. The defecting force is in axial symmetry, so the focus (for the left-handed helix) or defocus (for the right-handed helix) effects are in transverse directions as shown in Fig. 2.

To evaluate how many radiations from the electrons in transverse phase space are monochromatic, the radiation phase error is calculated from electron trajectory. As shown in Fig. 3, the BLU is sensitive to the transverse place where electron passes through, however it is tolerant to slope the angle of the electron. It also shows that a monochromatic radiation is produced with the transverse acceptance of just around 2 microns, which requires a novel focus lattice design for the electron beam at the end of the linac [28].

The photon flux and brightness are of the most concern for the light sources. The flux produced by the BLU is evaluated by SPECTRA [29] in this paper. The electron beam parameters are listed in Table II and the Bessel light beam parameters are as in Table I. We assume electron beam size could be tightly focused to $1 \mu \mathrm{m}$ to radiate

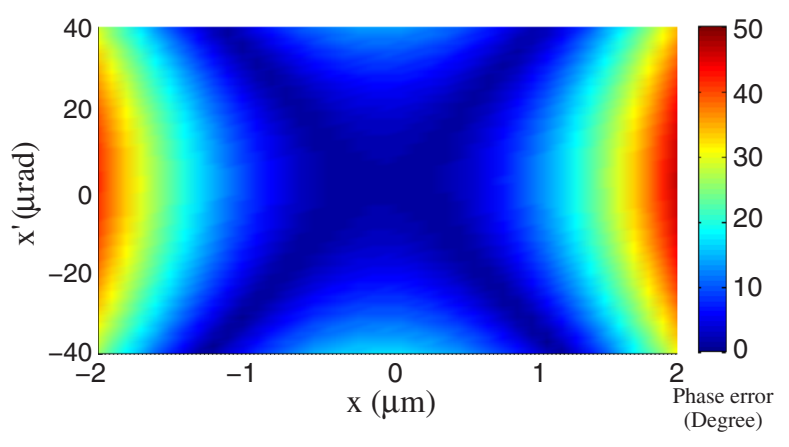

FIG. 3. Radiation phase errors for electrons with different initial $x-x^{\prime}$ conditions. monochromatically; the flux can exceed $10^{6}$ photons in $0.1 \%$ bandwidth per pulse. For a normally focused electron beam, beam size at $10 \mu \mathrm{m}$, the flux is 2 orders lower as shown in Table II.

For comparing, LCS photon flux is simulated using CAIN [30]. The cross angle of the electron beam to the laser is 51 degrees which is calculated by the formula in [25] to produce flux peak around $10 \mathrm{keV}$. The laser parameters are also listed in Table II. The electron beam parameters are the same as for BLU with one beam size $1 \mu \mathrm{m}$ and the other $10 \mu \mathrm{m}$. The flux is on the order of $10^{4}$ photons in $0.1 \%$ bandwidth.

The advantages of BLU in terms of flux is not obvious. However for brightness, it will be much higher. BLU produced radiation is in the cone of $0.066 \mathrm{mrad}$, however LCS radiation is in cone $2.5 \mathrm{mrad}$. Thus BLU radiation brightness will get 2 to 3 orders higher compared to LCS.

TABLE II. Flux comparing between BLU and LCS.

\begin{tabular}{|c|c|}
\hline \multicolumn{2}{|l|}{ Electron beam } \\
\hline Energy & $0.84 \mathrm{GeV}$ \\
\hline Bunch charge & $1 \mathrm{nC}$ \\
\hline Bunch length & $1 \mathrm{ps}$ \\
\hline Normalized emittance & $2 \mathrm{~mm} \cdot \mathrm{mrad}$ \\
\hline Laser for LCS & \\
\hline Pulse energy & $30 \mathrm{~J}$ \\
\hline Pulse length & $3 \mathrm{ps}$ \\
\hline Laser wavelength & $10.6 \mu \mathrm{m}$ \\
\hline Spot size & $100 \mu \mathrm{m}$ \\
\hline BLU flux & \\
\hline $\begin{array}{l}\text { Flux @ } 10.11 \mathrm{KeV} \\
@ \text { e-beam size } 1 \mu \mathrm{m}\end{array}$ & $1.34 \times 10^{6}$ photons $/ 0.1 \%$ B.W./pulse \\
\hline $\begin{array}{l}\text { Flux @ } 10.11 \mathrm{KeV} \\
\quad @ \text { e-beam size } 10 \mu \mathrm{m}\end{array}$ & $1.55 \times 10^{4}$ photons $/ 0.1 \%$ B.W./pulse \\
\hline $\begin{array}{l}\text { Photon divergence (rms) } \\
\text { LCS flux }\end{array}$ & $0.066 \mathrm{mrad}$ \\
\hline $\begin{array}{l}\text { Flux @ } 10.11 \mathrm{KeV} \\
\quad @ \text { e-beam size } 1 \mu \mathrm{m}\end{array}$ & $3.12 \times 10^{4}$ photons $/ 0.1 \%$ B.W./pulse \\
\hline $\begin{array}{l}\text { Flux @ } 10.11 \mathrm{KeV} \\
\quad @ \text { e-beam size } 10 \mu \mathrm{m}\end{array}$ & $4.07 \times 10^{4}$ photons $/ 0.1 \%$ B.W./pulse \\
\hline Photon divergence (rms) & $2.5 \mathrm{mrad}$ \\
\hline
\end{tabular}


The reason BLU holds a small radiation cone is that photons produced by each period of BLU can superimpose in a right phase at the one-axis direction which makes the photons in a small radiation cone and the spectrum holds narrow peaks. While for LCS, the produced photons are incoherent and get a wide range of radiation directions corresponding to different photon energies.

\section{DISCUSSIONS}

The BLU has unique properties that other types of undulators do not have. It only undulates a part of the electrons when the laser pulse is shorter than the electron beam. Because the Bessel light beam is at the speed of light, the interaction is limited at the place where electrons and the laser overlap in the longitudinal coordinate. This property can be used for short pulse (femtosecond) x-ray production with picosecond electron beam bunch.

The undulated electrons may shift backward in the longitudinal coordinate, as the interaction is located in a part of the longitudinal area of the electron beam, which may cause an electron beam density modulation.

From the preliminary tracking results, it can be found that BLU may radially focus (or defocus) electrons, which is different to quadrupoles and solenoids. More research efforts are needed for applications of the effects.

\section{ACKNOWLEDGMENTS}

The authors thank Professor Alex Chao greatly for useful discussions. Thanks also go to Professor Qiaogen Zhou for discussion on undulator performances and Professor Sheng Kanglong for language polishing. The work is supported by the National Key Research and Development Program of China (2016YFA0402001).

[1] J. Schwinger, On the classical radiation of accelerated electrons, Phys. Rev. 75, 1912 (1949).

[2] K.-Je Kim, Characteristics of synchrotron radiation, AIP Conf. Proc. 184, 565 (1989).

[3] Laser handbook, edited by W. B. Colson, C. Pellegrini, and A. Reniery (Elsevier Science Publisher B.V., Amsterdam, 1990), Vol. 6.

[4] W. Gudat, J. Pflüger, J. Chatzipetros, and W. Peatman, An undulator/multipole wiggler for the BESSY storage ring, Nucl. Instrum. Methods Phys. Res., Sect. A 246, 50 (1986).

[5] S. Yamamoto, T. Shioya, M. Hara, H. Kitamura, X. W. Zhang, T. Mochizuki, H. Sugiyama, and M. Ando, Construction of an in-vacuum type undulator for production of undulator $\mathrm{x}$ rays in the $5-25 \mathrm{keV}$ region, Rev. Sci. Instrum. 63, 400 (1992).

[6] T. Hara, T. Tanaka, H. Kitamura, T. Bizen, X. Maréchal, T. Seike, T. Kohda, and Y. Matsuura, Cryogenic permanent magnet undulators, Phys. Rev. ST Accel. Beams 7, 050702 (2004).
[7] J. Chavanne et al., in Proceedings of the 11th European Particle Accelerator Conference, Genoa, 2008 (EPS-AG, Genoa, Italy, 2008), pp. 2243-2245.

[8] S. Casalbuoni, T. Baumbach, S. Gerstl, A. Grau, M. Hagelstein, T. Holubek, D. Saez de Jauregui, C. Boffo, and W. Walter, Development of superconducting undulators at ANKA, Synchrotron Radiat. News 24, 14 (2011).

[9] Y. Ivanyushenkov et al., in Proceedings of the 24th Particle Accelerator Conference, PAC-2011, New York, 2011 (IEEE, New York, 2011), p. 1286.

[10] J. Clarke, The Science and Technology of Undulators and Wigglers (Oxford University Press, New York, 2004).

[11] T. Shintake, K. Huke, J. Tanaka, I. Sato, and I. Kumabe, Development of microwave undulator, Jpn. J. Appl. Phys. 22, 844 (1983).

[12] S. Tantawi, M. Shumail, J. Neilson, G. Bowden, C. Chang, E. Hemsing, and M. Dunning, Experimental Demonstration of a Tunable Microwave Undulator, Phys. Rev. Lett. 112, 164802 (2014).

[13] M. Zolotorev, Laser driven attosecond SASE X-ray FEL, Nucl. Instrum. Methods Phys. Res., Sect. A 483, 445 (2002).

[14] A. Bacci, M. Ferrario, C. Maroli, V. Petrillo, and L. Serafini, Transverse effects in the production of $\mathrm{x}$ rays with a free-electron laser based on an optical undulator, Phys. Rev. ST Accel. Beams 9, 060704 (2006).

[15] P. Sprangle, B. Hafizi, and J. R. Penano, Laser-pumped coherent x-ray free-electron laser, Phys. Rev. ST Accel. Beams 12, 050702 (2009).

[16] S. G. Rykovanov, C. B. Schroeder, E. Esarey, C. G. R. Geddes, and W. P. Leemans, Plasma Undulator Based on Laser Excitation of Wakefields in a Plasma Channel, Phys. Rev. Lett. 114, 145003 (2015).

[17] S. G. Rykovanov, J. W. Wang, V. Yu. Kharin, B. Lei, C. B. Schroeder, C. G. R. Geddes, E. Esarey, and W. P. Leemans, Tunable polarization plasma channel undulator for narrow bandwidth photon emission, Phys. Rev. ST Accel. Beams 19, 090703 (2016).

[18] L. Allen, M. W. Beijersbergen, R. J. C. Spreeuw, and J. P. Woerdman, Orbital angular momentum of light and the transformation of Laguerre-Gaussian laser modes, Phys. Rev. A 45, 8185 (1992).

[19] M. W. Beijersbergen, L. Allen, H. E. L. O. van der Veen, and J. P. Woerdman, Astigmatic laser mode converters and transfer of orbital angular momentum, Opt. Commun. 96, 123 (1993).

[20] V. H. Mellado, S. Hacyan, and R. Jauregui, Trapping and acceleration of charged particles in Bessel beams, Laser Part. Beams 24, 559 (2006).

[21] I. Bialynicki-Birula, Z. Bialynicka-Birula, and B. Chmura, Trojan states of electrons guided by bessel beams, Laser Phys. 15, 1371 (2005).

[22] B. Hafizi, E. Esarey, and P. Sprangle, Laser-driven acceleration with Bessel beams, Phys. Rev. E 55, 3539 (1997).

[23] Y. Shi, B. Shen, L. Zhang, X. Zhang, W. Wang, and Z. Xu, Light Fan Driven by a Relativistic Laser Pulse, Phys. Rev. Lett. 112, 235001 (2014).

[24] J. Durnin and J. J. Miceli, Jr., and J. H. Eberly, DiffractionFree Beams, Phys. Rev. Lett. 58, 1499 (1987). 
[25] G. A. Krafft and G. Priebe, Compton sources of electromagnetic radiation, Rev. Accel. Sci. Techol. 03, 147 (2010).

[26] D. L. Andrews and M. Babiker, The Angular Momentum of Light (Cambridge University Press, Cambridge, England, 2013).

[27] Z. T. Zhao and D. Wang, in Proceedings of the 32nd Free Electron Laser Conference, Malmö, Sweden (Max-lab, Sweden, 2010), p. 15.
[28] P. Raimondi and A. Seryi Novel Final Focus Design for Future Linear Colliders, Phys. Rev. Lett. 86, 3779 (2001).

[29] T. Tanaka and H. Kitamura, SPECTRA: a synchrotron radiation calculation code, J. Synchrotron Radiat. 8, 1221 (2001).

[30] K. Yokoya, User's Manual of CAIN, https://ilc.kek.jp/ yokoya/CAIN/. 